\title{
BOLL WEEVIL
}

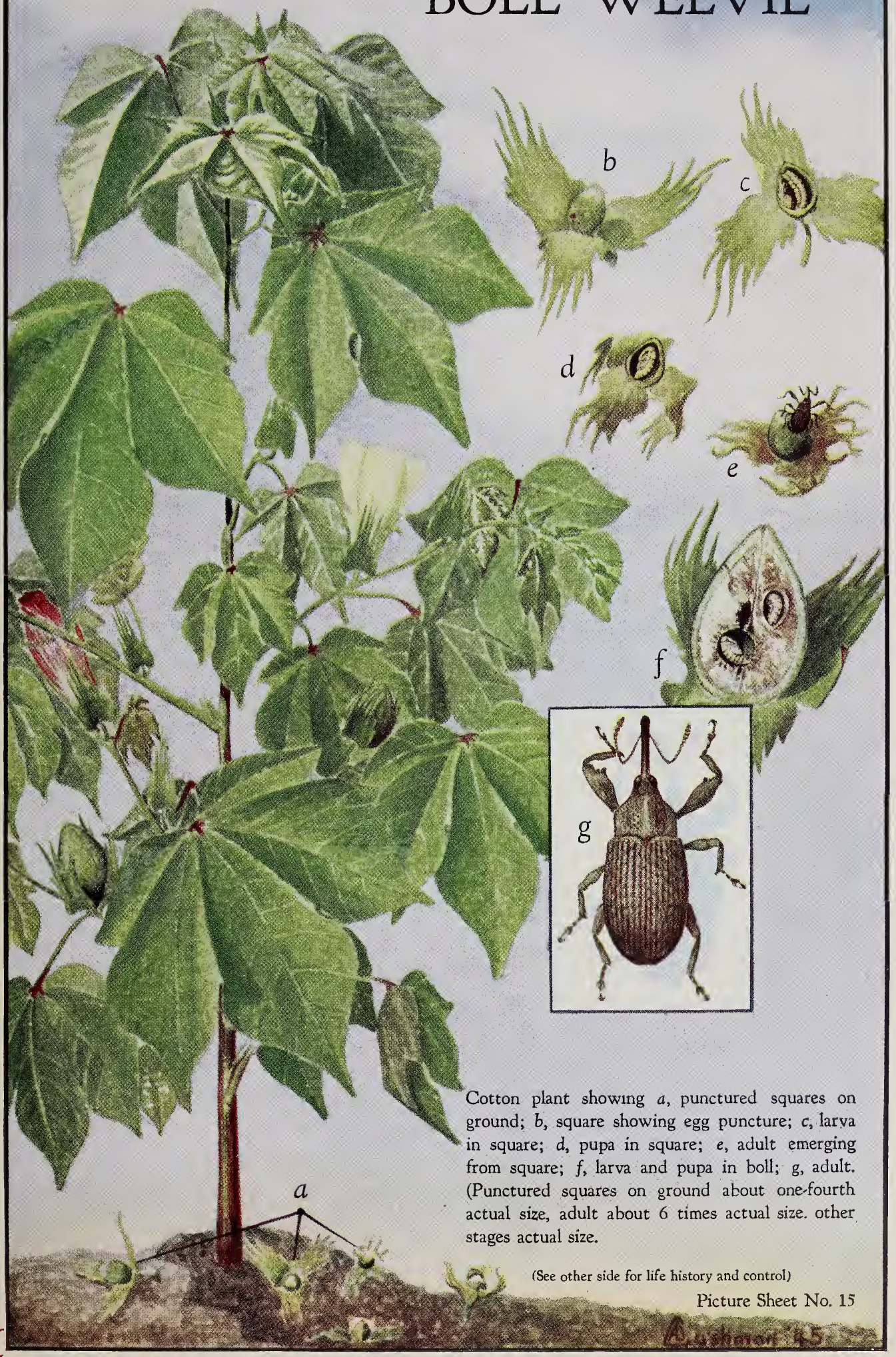

466 Bureau of Entomology and Plant Quarantine, Agricultural Research Administration, United States Department of Agriculture 452 


\section{BOLL WEEVIL}

\section{Life History}

Boll weevils pass the winter as adults in weeds, grass, woods trash, or other protected places near cottonfields. They leave winter quarters and return to cottonfields in the spring when the weather is warm enough for cotton to grow, and they remain there until frost. Boll weevils prefer to feed on and to lay their eggs in squares, but they also attack bolls. Eggs are laid singly in deep punctures made within the squares or bolls, and after 3 to 5 days they hatch into white larvae, or grubs. The grubs feed for 7 to 14 days and then change into pupae within the squares or bolls. The adults emerge from the pupae in 3 to 5 days and cut their way out. After feeding on blooms, squares, or bolls for 3 to 4 days, the females are ready to lay eggs. The complete life cycle from egg to adult weevil requires about 3 weeks when temperatures are high, and there may be seven or eight generations a season.

The leaflike bracts at the base of the punctured squares open up, or flare, and the square turns yellow and dies. Most of the punctured squares and small bolls are shed, but some remain hanging to the plants. Large punctured bolls are not shed, but the lock in which a grub feeds fails to develop properly, and the lint is cut, stained brown, and decayed. When several weevil grubs develop within a boll, as often occurs, the entire boll is ruined.

\section{Control \\ Cultural Control}

Farming practices that help set bolls quickly will aid in weevil control. These practices are as follows :

1. Plant cotton on good land that has been well prepared.

2. Use fertilizer recommended for your locality.

3. Select an early maturing variety suited for growing in your section.

4. Plant early, space closely, and cultivate frequently.

5. Pick early and cleanly. After the cotton has been picked, stop further fruiting by plowing out, cutting, or grazing the cotton stalks as early as possible in the fall, to reduce the number of weevils in next year's crop.

\section{Control with Dusts}

Dusting with calcium arsenate during the time cotton is fruiting is the most practicable method of direct control. To determine when dusting is needed, examine squares once a week or oftener for weevil punctures. Walk diagonally across the field and pick 100 squares that are half-grown or larger. Pull only 1 square from a plant, taking about the same number from topmost, middle, and lowest branches. When 100 squares have been picked, count those having weevil punctures to determine the percentage of infestation. On light soils where cotton does not grow rank and matures early, dusting should begin when 10 to 15 percent of the squares are punctured. On fertile soils where cotton continues to grow and fruit until late in the season, do not begin dusting until 20 to 25 percent of the squares are punctured.

Dust with 5 to 7 pounds of calcium arsenate per acre every 4 or 5 days until the weevils are brought under control, or until a crop of bolls is set. It is advisable to mix 1 or 2 percent of nicotine with the calcium arsenate to prevent aphid damage. Repeat if the dust is washed off by rain within 24 hours. From three to five applications will usually control weevils and result in the setting of a crop. From one to three dustings are sometimes needed to protect bolls.

Dusting may be done early in the morning, late in the afternoon, or at night, when the air is quiet. It is more important to dust when the air is calm than when the plants are wet with dew.

Low winter temperatures and hot, dry summers help control the boll weevil. Watch for a rapid increase of weevils and severe damage during rainy periods.

Plant lice, or aphids, can be held in check by adding nicotine to the calcium arsenate. Nicotine should not be applied during midday, when the dust has a tendency to rise. For effective aphid control it is necessary that the dust containing nicotine stay down among the plants, not rise and float away.

Caution: Calcium arsenate is a poison and should be handled with care. Store it in a dry place where children and animals will not have access to it.

April 1946

U. S. Government Printing Office

For sale by the Superintendent of Documents, Washington 25, D. C. Price 5 cents $684389-46$ 